\title{
NANOPARTÍCULAS COMO TRANSPORTADORES DE FÁRMACOS: UNA HERRAMIENTA PROMETEDORA CONTRA LA TUBERCULOSIS
}

\author{
Julieta Luna-Herrera@1,a, Damián Eduardo Pérez-Martínez@i(b,c, \\ Virginia Margarita Barradas-Hernández ${ }^{3, \mathrm{~d}, \mathrm{~d}}$, Roberto Zenteno-Cuevas $\mathbb{1}^{3, \mathrm{~b}, \mathrm{e}}$ \\ ${ }^{1}$ Departamento de Inmunología, Escuela Nacional de Ciencias Biológicas, Instituto Politécnico Nacional, Ciudad de \\ México, México. \\ 2 Instituto de Ciencias de la Salud, Universidad Veracruzana, Xalapa, Veracruz, México. \\ 3 Instituto de Salud Pública, Universidad Veracruzana, Xalapa, Veracruz, México. \\ a Química bacterióloga parasitóloga, doctora en Inmunología; ${ }^{\mathrm{b}}$ biólogo; ${ }^{\mathrm{c}}$ maestro en Salud Pública; ${ }^{\mathrm{d}}$ médico cirujano; \\ e doctor en Ciencias.
}

\section{RESUMEN}

La Organización Mundial de la Salud (OMS) ubica a la tuberculosis (TB) como uno de los problemas de salud más preocupantes en la actualidad, y señala que se requieren de acciones novedosas para controlar su expansión y, de esta manera, alcanzar una de las metas establecidas en los Objetivos de Desarrollo Sostenible: reducir para 2030 la morbilidad e incidencia de TB. Para lograr este objetivo, está claro que las herramientas empleadas actualmente para su diagnóstico y tratamiento ya no son las adecuadas. En este sentido, es necesario desarrollar nuevos medicamentos y vacunas, así como novedosos procedimientos de administración de fármacos que generen una mejor respuesta, disminuyan el tiempo y optimicen los tratamientos. La nanotecnología ha incorporado en los últimos años un gran número de nuevas herramientas que incrementan considerablemente, la diversidad de mecanismos para la administración de tratamientos antituberculosos. Dicho esto, la presente revisión describe brevemente el estado actual de la farmacorresistencia en TB, así como las características generales de las nanopartículas que están evaluándose como herramientas para transportar antibióticos antituberculosos.

Palabras clave: Tuberculosis; Nanopartículas; Farmacorresistencia Microbiana; Antiinfecciosos; Transporte Biológico (fuente: DeCS BIREME).

\section{NANOPARTICLES AS DRUG TRANSPORTERS: A PROMISING TOOL AGAINST TUBERCULOSIS}

Citar como: Luna-Herrera J, Pérez-Martínez DE, BarradasHernández VM, ZentenoCuevas R. Nanopartículas como transportadores de fármacos: una herramienta prometedora contra la tuberculosis. Rev Peru Med Exp Salud Publica. 2021;38(1):14352. doi: https://doi.org/10.17843/ rpmesp.2021.381.6156.

Correspondencia: Roberto ZentenoCuevas; Instituto de Salud Pública, Universidad Veracruzana. Av. Luis Castelazo Ayala s/n, A.P. 57. Col. Industrial Animas. Xalapa, Veracruz, 91190, México; robzencue@gmail.com

Recibido: $13 / 07 / 2020$ Aprobado: 30/09/2020 En línea: 01/02/2021

\section{ABSTRACT}

The World Health Organization (WHO) places Tuberculosis (TB) as one of the most important health problems today. According to the WHO, this disease requires novel actions to control its expansion and, in this way, achieve one of the goals established in the sustainable development goals: to reduce TB morbidity and incidence by 2030 and regain control. To achieve this goal, the tools currently used for diagnosis and treatment are no longer adequate. In this sense, it is necessary to develop new drugs and vaccines, as well as novel drug administration procedures that generate a better response, reduce times, and optimize treatments. Nanotechnology has incorporated in recent years a considerable number of new tools that significantly increase the diversity of mechanisms for the administration of anti-tuberculosis drugs. Therefore, the present review briefly describes the current state of drug resistance in tuberculosis, as well as the general characteristics of nanoparticles being evaluated as tools to transport new antibiotics against tuberculosis.

Keywords: Tuberculosis; Nanoparticles; Antimicrobial Drug Resistance; Anti-Infective Agents; Biological Transport (source: MeSH NLM).

\section{INTRODUCCIÓN}

En el informe anual de la Organización Mundial de la Salud (OMS) del 2018 se reportaron 10,5 millones de nuevos casos de tuberculosis (TB) y 1,5 millones de muertes por esta enfermedad, estas cifras ubican a la TB como la enfermedad infecciosa más grave del mundo ${ }^{(1)}$. Asimismo, cerca de 200000 casos de TB presentaron algún tipo de resistencia y 161000 mostraron resistencia a la rifampicina ${ }^{(1)}$. Estas cifras revelan la crítica situación de la resistencia a los medicamentos y confirman la urgente necesidad de incluir nuevos y más eficaces fármacos, mecanismos de 
administración y tratamiento. En respuesta, una nueva generación de fármacos contra $\mathrm{TB}$ está emergiendo, por ejemplo, bedaquilina, delamanid, pretomanid y linezolid.

Sin embargo, la farmacorresistencia a la TB se mantiene como una seria amenaza para la humanidad; por lo que es urgente desarrollar acciones que reduzcan su impacto. Dentro de estas acciones es necesario aprovechar de manera adecuada los fármacos que se conocen y usan hasta hoy. Considerando lo anterior, la nanotecnología y el desarrollo de nanopartículas se han posicionado como elementos que puedan aportar beneficios en el tratamiento de la TB y lograr la disminución de la resistencia. A partir de lo anterior, en esta revisión se pretende revisar las nanopartículas que se emplean como transportadoras de fármacos antituberculosos.

\section{BÚSQUEDA Y SELECCIÓN DE EVIDENCIA}

Se realizó una búsqueda en la base de datos PubMed hasta el 15 de febrero de 2020. Se incluyeron reportes de investigación y algunas revisiones, además de reportes oficiales de la OMS. La búsqueda de términos incluyó las palabras clave y los conectores: “Tuberculosis"[All Fields] AND "Nanoparticles"[All Fields]. Se usaron filtros para recuperar artículos en inglés y a texto completo.

Se encontraron 621 artículos relacionados al tema, se seleccionaron preferentemente aquellos documentos publicados en los últimos trece años. Con este segundo filtro se seleccionaron 135 artículos, de los cuales se realizó una depuración final de acuerdo con el impacto, la trascendencia y la originalidad del estudio.

\section{LA TUBERCULOSIS COMO UN PROBLEMA DE SALUD PÚBLICA}

La TB es una enfermedad infecciosa causada principalmente por Mycobacterium tuberculosis y se trasmite de persona a persona a través de gotas liberadas por el individuo infectado. El diagnóstico de la TB se realiza principalmente mediante baciloscopía, además existen otros procedimientos como la radiografía de tórax y el cultivo de aislado clínico de micobacteria. Estas tres técnicas han sido empleadas por décadas, sin embargo, sus porcentajes de sensibilidad y especificidad oscilan entre el $60 \%$ y el $90 \%$, por lo cual se requieren de horas o hasta semanas para obtener resultados. Debido a estas características, se considera que estos métodos no pueden emplearse como herramientas de apoyo para controlar y erradicar la TB de la lista de problemas de salud para el $2030^{(2)}$.

Como respuesta a lo anterior, en los últimos años se ha desarrollado una nueva generación de procedimientos de diagnóstico molecular, basados en el análisis de ADN de TB. Si bien existe una gran diversidad, la OMS solo ha avalado algunos de estos procedimientos, como: la amplificación isotérmica de TB mediada por loop mediated isothermal amplification), prueba de sonda en línea (Line Probe Assays), GeneXpert, y muy recientemente la secuenciación del genoma completo (Whole Genome Sequencing) ${ }^{(3)}$. Estos sistemas se caracterizan por tener una capacidad diagnostica dual, por un lado, confirman la presencia del agente infeccioso y por otro identifican mutaciones causantes de la resistencia a fármacos de primera y segunda línea, con niveles de sensibilidad y especificidad superiores al $80 \%-90 \%$, por lo que estas pruebas están imprimiendo una dinámica diagnóstica sin precedentes en el estudio de la $\mathrm{TB}^{(3)}$.

La TB es una enfermedad curable, cuyo esquema de tratamiento es conocido como tratamiento acortado estrictamente supervisado e implica el uso de cuatro antibióticos: isoniacida $(\mathrm{H})$, rifampicina $(\mathrm{R})$, pirazinamida $(\mathrm{Z})$ y etambutol (E), en un período de cuatro a seis meses. Si no se observa supresión de la infección, se determina entonces el perfil de resistencia a fármacos de primera línea y se administra un nuevo grupo de medicamentos ubicados dentro del esquema de segunda línea (Tabla suplementaria 1) ${ }^{(4)}$.

La administración de regímenes farmacológicos inadecuados, la mala adherencia o el abandono del tratamiento, el virus de inmunodeficiencia humana (VIH) y la diabetes mellitus de tipo 2, son factores que se encuentran asociados al desarrollo de la TB farmacorresistente. La OMS ha clasificado la farmacorresistencia de la TB en cuatro tipos: 1) monorresistente, resistencia a solo un fármaco; 2) polirresistente, resistencia simultánea a dos o más fármacos, que no incluyan la combinación H y R; 3) multidrogorresistente (MDR-TB), resistencia simultánea a $\mathrm{H}$ y $\mathrm{R}$ y/o cualquier otro fármaco; y 4) extremo farmacorresistente (XDR-TB), es MDR-TB y además es resistente a una fluoroquinolona y a algún fármaco inyectable de segunda línea ${ }^{(4)}$.

De acuerdo con el informe global de TB del 2018, de los diez millones de casos nuevos reportados en 2017, cerca de 200000 presentaron algún tipo de resistencia, 161000 mostraron resistencia a la rifampicina y cerca de 14000 fueron XDR-TB ${ }^{(1)}$. Estas cifras revelan la situación crítica de la resistencia a los medicamentos y confirman la necesidad urgente de incluir nuevos y más eficaces medicamentos y tratamientos para que la TB deje de ser un problema de salud pública mundial para el $2030^{(4)}$. En respuesta, una nueva generación de medicamentos contra la TB está emergiendo: bedaquilina, delamanid, pretomanid y linezolid son solo algunos de ellos; sin embargo, se están empezando a describir reportes en los que se identifican aislados con resistencia hacia estos fármacos ${ }^{(5)}$. 


\section{LA FARMACORRESISTENCIA COMO UN GRAVE PROBLEMA DE SALUD PARA EL 2050}

La farmacorresistencia es una seria amenaza para la humanidad. Se ha estimado que si no se toman acciones desde ahora para controlar infecciones causadas por bacterias resistentes, como Mycobacterium tuberculosis, Staphylococcus aureus, Pseudomonas aeruginosa, Neisseria gonorrhoeae, etc., el número de muertes para el 2050 superará las diez millones de personas anualmente, lo cual supera las defunciones provocadas por enfermedades como el cáncer o la diabetes ${ }^{(6,7)}$. Estas proyecciones igualmente señalan que es urgente desarrollar acciones para disminuir el incremento en la resistencia bacteriana. Dentro de estas acciones se consideran como prioritarias mejorar el diagnóstico, aprovechar los fármacos que se usan en la actualidad, reposicionar los fármacos que dejaron de usarse e incrementar la inversión de recursos y desarrollo de nuevas moléculas dirigidas a nuevos blancos terapéuticos ${ }^{(7)}$.

Considerando lo anterior, la nanotecnología puede aportar grandes beneficios en la disminución de la resistencia a los fármacos no solo para la TB, sino en general para cualquier infección. En el área de diagnóstico, la utilización de la nanotecnología podría igualmente contribuir en la innovación de los procedimientos de detección oportuna de enfermedades infecciosas ${ }^{(8)}$; y en el área de innovación farmacéutica, en la mejora de los sistemas de liberación de fármacos y su optimización para reducir los tiempos de tratamiento, que usualmente son largos.

\section{LA NANOTECNOLOGÍA Y EL CUMPLIMIENTO DE LOS OBJETIVOS DE DESARROLLO SOSTENIBLE RELACIONADOS CON LA TUBERCULOSIS}

De los 13 objetivos de desarrollo sostenible, adoptados por las ONU en 2015, el punto 3.3 menciona específicamente el poner fin a las epidemias de TB para el 2030, lo cual reduciría el $90 \%$ de las muertes y el $80 \%$ de la incidencia. Uno de los elementos que sustenta dicho objetivo establece que «se intensificará la investigación y la innovación, centrándose en el descubrimiento, el desarrollo y la rápida adopción de nuevos instrumentos, intervenciones y estrategias. Asimismo, investigar para optimizar la aplicación, el impacto y promover las innovaciones» ${ }^{(2)}$.

En este sentido, la nanotecnología y el desarrollo de nanomateriales son áreas de investigación y desarrollo tecnológico que en los últimos años están presentando un importante número de aportes al estudio de la TB ${ }^{(8-11)}$. Dentro de las diver- sas ramas de la nanotecnología, una de las más activas es el desarrollo, la creación y uso de nanopartículas (NP), las cuales son partículas de 10-100 nm de tamaño y en casos excepcionales hasta $1000 \mathrm{~nm}$. Constituidas por un número diverso de moléculas, que conforman estructuras sumamente útiles para transportar una amplia gama de moléculas, por lo que poseen grandes implicancias en varios campos, incluso en la farmacia y la medicina. Esta propiedad de transporte se ha aprovechado en el escenario de TB y se utiliza para enviar antígenos vacunales y fármacos, entre otros ${ }^{(12)}$.

Las NP y los nanomateriales tienen diferentes propiedades estructurales y biológicas, modificables según los polímeros, las soluciones y los aditivos utilizados para su fabricación. Sus composiciones les confieren diversas propiedades, como la protección del contenido, el incremento de su solubilidad, la absorción y la biodisponibilidad de las moléculas transportadas. Además, pueden ser modificables para orientar la captación de las NP a un tipo específico de blanco celular o tisular, incrementando su eficiencia. Finalmente, es importante mencionar que poseen una gran capacidad para ser administradas de diferentes maneras, como vía oral, tópica, parenteral $\mathrm{y}$ mucosa por lo que tienen una gran versatilidad ${ }^{(8-1)}$.

Actualmente, las NP y los nanomateriales diseñados específicamente para administrar medicamentos se clasifican en cinco grandes grupos: 1) NP fundamentadas en el empleo de lípidos, 2) NP constituidas por emulsiones, 3) NP constituidas en vesículas, 4) NP conformadas por estructuras misceláneas, y 5) nanofibras ${ }^{(9,11)}$.

A continuación, se hace una descripción de las características más importantes de estas NP y como están siendo evaluadas para emplearse como tecnologías emergentes para la administración de fármacos en contra de la TB.

\section{NANOPARTÍCULAS BASADAS EN LÍPIDOS}

\section{Micropartículas de lípidos sólidos (M-LS)}

Son partículas coloidales con un tamaño que ronda entre los 10-1000 nm, están compuestas por lípidos biodegradables fundidos en agua o en una solución acuosa. Las M-LS poseen una matriz central conformada por un núcleo lipídico sólido, que puede solubilizar y estabilizar medicamentos o moléculas lipofílicas. Su éxito en la administración de fármacos se debe a su capacidad de atravesar barreras anatómicas de manera eficiente y a la posibilidad de incluir dos fármacos con diferentes características, uno lipofílico y otro hidrofílico. Además, de acuerdo con diversos estudios, poseen una alta biodisponibilidad y baja toxicidad al utilizarse como tratamiento antituberculoso. En la Tabla 1, se muestran algunos ejemplos del uso de M-LS para transportar fármacos antituberculosos ${ }^{(13-17)}$. 
Tabla 1. Nanopartículas basadas en lípidos, lípidos-sólidos y lipídicas nanoestructuradas usadas como transportadores de fármacos antituberculosos*

\begin{tabular}{|c|c|c|c|c|}
\hline Fármaco & VA & In vitro $/ \mathrm{PC}$ & Comentarios & Ref. \\
\hline \multicolumn{5}{|c|}{ Micro y macropartículas de lípidos sólidos (M-LS) (Figura 1A) } \\
\hline $\mathrm{R}$ & Oral & $+/+$ & $\begin{array}{l}\text { Se aprecia una mayor biodisponibilidad oral del fár- } \\
\text { maco después de la aplicación de la NP. La concen- } \\
\text { tración mínima inhibitoria del fármaco en plasma } \\
\text { se mantuvo constante durante cinco días. }\end{array}$ & 15 \\
\hline $\mathrm{R}$ & Inhalatoria & $+1-$ & $\begin{array}{l}\text { Alta eficiencia de unión a mucina. Mayor eficiencia } \\
\text { de absorción in vitro de las NP acopladas a macró- } \\
\text { fagos. }\end{array}$ & 16 \\
\hline $\mathrm{R}$ & Inhalatoria & $+/-$ & $\begin{array}{l}\text { Aumento de captación in vitro de NP (superficie de } \\
\text { metil } \alpha \text {-D-mannopiranoside) por macrófagos mu- } \\
\text { rinos. }\end{array}$ & 17 \\
\hline $\mathrm{H}$ & ND & $+/-$ & $\begin{array}{l}\text { Las NP lipídicas sólidas funcionales por manosa } \\
\text { son efectivas para atacar macrófagos alveolares. }\end{array}$ & 18 \\
\hline $\mathrm{R}$ & ND & $+/-$ & $\begin{array}{l}\text { Las NP cargadas de R se absorbieron selectivamente } \\
\text { mejor en los macrófagos alveolares que en los del } \\
\text { epitelio alveolar. }\end{array}$ & 19 \\
\hline \multicolumn{5}{|c|}{ Nanopartículas de lípidos sólidos polímeros (N-LSP) (Figura 1A) } \\
\hline Cfx & Inhalatoria & $+/+$ & $\begin{array}{l}\text { Se presentó una deposición pulmonar de las NP a } \\
\text { niveles de mayor profundidad. Más de la mitad de } \\
\text { la dosis se localizó en los pulmones después de } 30 \\
\text { minutos y con una acumulación importante duran- } \\
\text { te } 24 \text { horas. }\end{array}$ & 20 \\
\hline \multicolumn{5}{|c|}{ Nanopartículas con lípidos nano estructurados (N-LNEs) (Figura 1A) } \\
\hline Bdq & Intravenosa & $+/-$ & $\begin{array}{l}\text { Los valores de concentración mínima inhibitoria } \\
\text { de Bdq no se modificaron después de su encapsula- } \\
\text { ción. No se observaron efectos citotóxicos en líneas } \\
\text { celulares estudiadas. }\end{array}$ & 21 \\
\hline $\mathrm{R}$ & ND & $+/+$ & $\begin{array}{l}\text { Las NP cargadas con el fármaco R mostraron se- } \\
\text { lectividad tisular y una acumulación pulmonar } \\
\text { importante. Estas NP mostraron una mejora en su } \\
\text { absorción en macrófagos alveolares. }\end{array}$ & 22 \\
\hline
\end{tabular}

* Las estructuras de estas nanopartículas se presenta en la Figura 1A.

VA: vía de administración; PC: preclínico; ND: sin datos; NP: nanopartículas; R: rifampicina; H: isoniazida; Cfx: ciprofloxacina; Bdq: bedaquilina.

\section{Nanopartículas de lípidos sólidos y polímeros (N-LSP)}

Contienen una concentración de hasta el 2,5\% de lípidos, como triglicéridos y ácidos grasos. Otros compuestos empleados en su estructura son la lecitina, óxido de etileno y propileno. Debido a los lípidos fisiológicos utilizados para su elaboración, estas estructuras tienen una alta tolerancia a la degradación, mayor estabilidad y mejor capacidad para incorporar sustancias hidrofóbicas o hidrofílicas sin efectos citotóxicos. El empleo de estas NP como trasportadoras de medicamentos contra la TB se pude apreciar en la Tabla $1^{(18)}$.

\section{Nanopartículas con lípidos nano estructurados (N-LNE)}

Estas NP son una alternativa a las M-LS, con la intención de obtener combinaciones de NP conformadas predominantemente por una matriz con lípidos sólidos y otro tipo de lípidos, en una proporción de 70:30. Gracias a esta mezcla es posible incorporar drogas lipofílicas e hidrofílicas que pueden administrarse vía oral, ocular, pulmonar e intravenosa.
La Tabla 1 muestra algunos reportes relacionados con el empleo de estas estructuras en el tratamiento de la $\mathrm{TB}^{(19,20)}$.

\section{NANOPARTÍCULAS BASADAS EN EMULSIONES}

\section{Nanopartículas de microemulsión (N-ME)}

Compuestas por agentes acuosos, oleosos y emulsificantes. Su diámetro oscila entre 10-100 nm. La composición y combinación de compuestos usados para su elaboración las hace termodinámicamente estables, incrementa la absorción, permeabilidad y protección de los fármacos transportados. Las $\mathrm{N}-\mathrm{ME}$ son útiles para administrar sustancias coloidales. Se reconocen tres tipos dependiendo de su composición basada en aceite en agua, bicontinuas y agua en aceite. La Tabla 2 indica algunos ejemplos de cómo están siendo evaluadas como transportadores de fármacos antituberculosos ${ }^{(21)}$.

\section{Nanopartículas de nanoemulsiones (N-NE)}

Son partículas con diámetros que van de 50 a 1000 nm. Poseen una estructura aceitosa y acuosa, y emplean cosurfac- 
Tabla 2. Características de las nanopartículas basadas en emulsiones utilizadas como transportadoras de fármacos antituberculosos*

\begin{tabular}{cccl}
\hline Fármaco & VA & In vitro/PC & \multicolumn{1}{c}{ Comentarios } \\
\hline R, H y Z & ND & ND & $\begin{array}{l}\text { La microemulsión se ha probado usando diferentes soluciones de } \\
\text { colorantes: el fármaco R mostró una fuerte asociación con el colo- } \\
\text { rante rojo del Nilo, mientras que los fármacos H y Z mostraron una } \\
\text { importante interacción con el di-cloruro de rutenio. }\end{array}$ \\
& ND $\begin{array}{ll}\text { Los medicamentos antituberculosos empleados conjuntamente con } \\
\text { esta NP mostraron un comportamiento de liberación real, con ex- } \\
\text { cepción del fármaco R. }\end{array}$ \\
\hline
\end{tabular}

* Las estructuras de estas nanopartículas se presenta en la Figura 1B.

VA: vía de administración; PC: preclínico; ND: sin datos; R: rifampicina; H: isoniazida; Z: pirazinamida; NP: nanopartícula.

tantes que generan una configuración termodinámica estable. La preparación de las N-NE puede hacerse fácilmente y con un bajo consumo de energía. Estas características ayudan a explicar por qué estas N-NE tienen un amplio espectro de uso en diversos campos de la salud, incluso en la evaluación de algunos ensayos para la administración de medicamentos antituberculosos. En la Tabla 2 se aprecian algunos ejemplos del uso de estas partículas ${ }^{(22)}$.

\section{NANOPARTIICULAS CONSTITUIDAS EN VESÍCULAS}

\section{Liposomas (LPS)}

Se trata de vesículas conformadas por una solución acuosa que se encuentra encerrada en una membrana lipídica, poseen un diámetro de entre $0,05 \mathrm{~nm}$ hasta $5,0 \mathrm{~nm}$, pueden ser fusionadas y procesadas creando estructuras con diferente tamaño, composición y carga. Los componentes lipídicos incluyen fosfolípidos y esfingolípidos, esta composición provee importantes ventajas biológicas, como biocompatibilidad, fácil degradación, baja o nula toxicidad e inmunogenicidad y habilidad de encapsular moléculas hidrofílicas y lipofílicas. Pueden ser internalizadas por células fagocíticas, fusionarse con lisosomas y degradarse, liberando su contenido dentro de las células. Esta capacidad es fundamental para administrar fármacos de forma eficiente contra patógenos intracelulares. Reportes sobre el empleo de estas partículas contra la TB se encuentran en la Tabla $3^{(23-27)}$.

\section{Niosomas (NIO)}

Son vesículas conformadas por una membrana bicapa lipídica, capaz de encapsular medicamentos en una membrana vesicular lipídica, similar a los liposomas, con las ventajas de que los materiales requeridos para su fabricación son más económicos,

Tabla 3. Nanopartículas vesiculares utilizadas como transportadoras de fármacos antituberculosos *.

\begin{tabular}{|c|c|c|c|c|}
\hline Fármaco & VA & In vitro /PC & Comentarios & Ref. \\
\hline \multicolumn{5}{|c|}{ Liposomas (LPS) (Figura 1C) } \\
\hline $\mathrm{R}$ & Inhalatoria & $+/-$ & $\begin{array}{l}\text { La NP reduce la toxicidad de R. El empleo de liposomas constituidos por } \\
\text { altas concentraciones de fosfolípidos y colesterol presentaron un mejor } \\
\text { encapsulamiento y captación por el tejido pulmonar. }\end{array}$ & 25 \\
\hline Car y Lfx & ND & $+/-$ & $\begin{array}{l}\text { Se observó una mejora del efecto fármaco Lfx en comparación con } \\
\text { el medicamento libre. }\end{array}$ & 26 \\
\hline $\mathrm{R}$ & Inhalatoria & $+/+$ & $\begin{array}{l}\text { Los liposomas usados en la administración inhalatoria mejoran la } \\
\text { permeabilidad del medicamento en el epitelio alveolar. }\end{array}$ & 27 \\
\hline $\mathrm{R}, \mathrm{H}, \mathrm{Z}$ y E & Inhalatoria & $+/+$ & Liposoma con liberación sostenida, prolongada y mejor absorción. & 28 \\
\hline Z & Inhalatoria & $+/+$ & Proliposomas cargados con eficiencia de encapsulación hasta del $45 \%$. & 29 \\
\hline \multicolumn{5}{|c|}{ Niosomas (NIO) (Figura 1C) } \\
\hline $\mathrm{Z}$ & Subcutánea & $+/+$ & $\begin{array}{l}\text { Se observó una disminución en la cantidad de micobacteria presen- } \\
\text { te en los pulmones de cerdos de Guinea. }\end{array}$ & 31 \\
\hline $\mathrm{E}$ & Subcutánea & $+/+$ & Se apreció una menor cantidad de micobacterias en los pulmones. & 30 \\
\hline $\mathrm{R}, \mathrm{H}$ y $\mathrm{Z}$ & Oral & $+/-$ & $\begin{array}{l}\text { Se observaron perfiles de liberación de H y Z bifásico. R con libera- } \\
\text { ción sostenida durante } 5 \mathrm{~h} \text {. }\end{array}$ & 32 \\
\hline \multicolumn{5}{|c|}{ Lipoesferas (LIP) (Figura 1D) } \\
\hline $\mathrm{R}$ & ND & $+/-$ & $\begin{array}{l}\text { Las lipoesferas cargadas con } \mathrm{R} \text { con un tamaño de } 247 \mathrm{~nm} \text { fueron } \\
\text { estables durante } 4 \text { semanas (conservación de } 4-25^{\circ} \mathrm{C} \text { ). }\end{array}$ & 34 \\
\hline
\end{tabular}

* Las estructuras de estas nanopartículas se presenta en la Figura $1 \mathrm{C}$ y $1 \mathrm{D}$.

VA: vía de administración; PC: preclínico; ND: sin datos; Car: cardiolipina; Lfx: levofloxacino; R: rifampicina; H: isoniazida; Z: pirazinamida; E: etambutol; NP: nanopartícula. 
estables y menos tóxicos, lo cual mejora el índice terapéutico del medicamento transportado. Además, estas NP pueden modificarse incorporando un receptor y dirigirse hacia células y tejidos específicos. Algunos ejemplos del empleo de esta NP en TB se pueden observar en la Tabla $3^{(28-31)}$.

\section{Lipoesferas (LIP)}

Son microesferas con un rango de $0,01 \mathrm{~nm}$ a $100 \mathrm{~nm}$ de diámetro. Se encuentran constituidas por lípidos predominantemente triglicéridos en los cuales se pueden incrustar fosfolípidos y otras moléculas similares. El núcleo se usa para transportar medicamentos con un carácter más hidrofóbico. Esta composición las hace ideales para que puedan administrarse vía oral, parenteral y tópica. Este tipo de NP se ha evaluado para transportar principalmente la rifampicina, algunos ejemplos de estas partículas se presentan en la Tabla $3^{(32)}$.

\section{NANOPARTÍCULAS CONFORMADAS POR ESTRUCTURAS MISCELÁNEAS}

\section{Dendrímeros (N-DNs)}

Son sistemas poliméricos altamente ramificados, con un diámetro de 2-10 $\mathrm{nm}$. Se fabrican con materiales sintéticos y pueden incluir grupos funcionales en la superficie que alteran su estructura y modifican su interacción con la célula o tejido blanco, así como su solubilidad, reactividad y miscibilidad. Estas estructuras tienen un amplio espacio vacío en su interior en el cual se pude encapsular el fármaco. Su toxicidad es baja y los grupos funcionales ayudan a mejorar y controlar la difusión de los medicamentos administrados. La Tabla 4 muestra ejemplos sobre el empleo de estas NP en la administración de fármacos en $\mathrm{TB}^{(33)}$.

\section{Nano/micro partículas (NMP)}

Formuladas con diferentes clases de polímeros, el ácido poli láctico coglicólico (PLGA) es el más común, mide entre 0,1 y $100 \mathrm{~nm}$. Las NMP pueden transformarse dependiendo de los surfactantes, solventes orgánicos y los fármacos utilizados para su elaboración. La conjugación con otras biomoléculas incrementa la vida media, la mucoadhesión y el reconocimiento de las células blanco. Debido a esta versatilidad biológica, las NMP tienen un efectivo sistema de liberación de fármacos que puede emplear diversas rutas de administración. En la Tabla 4 se aprecian reportes sobre el empleo de estas estructuras en contra de la $\mathrm{TB}^{(34-40)}$.

\section{Microesferas (N-Mcs)}

Partículas esféricas con diámetros de $1 \mathrm{~nm}$ a $1000 \mathrm{~nm}$ elaboradas con uno o más polímeros miscibles. Los medicamentos pueden dispersarse a un nivel micro o macroscópico. Se pueden construir empleando materiales naturales y sintéticos biodegradables. Se ha descrito el uso de N-Mcs para distribuir medicamentos antituberculosos vía plasma, en tejidos y órganos. Ejemplos del uso de este tipo de partículas para transportar fármacos antituberculosos se puede apreciar en la Tabla $5^{(41-43)}$.

\section{Nanosuspensión (NSP)}

Son NP sólidas dispersas en un vehículo acuoso formando un coloide. El tamaño de las partículas oscila entre $200 \mathrm{~nm}$ y $600 \mathrm{~nm}$. Estas estructuras se han empleado para transportar medicamen-

Tabla 4. Dendrímeros y nano/micropartículas utilizadas como transportadoras de fármacos antituberculosos*.

\begin{tabular}{|c|c|c|c|c|}
\hline Fármaco & VA & In vitro $/ \mathrm{PC}$ & Comentarios & Ref. \\
\hline \multicolumn{5}{|c|}{ Dendrímeros (N-DN) (Figura 1E) } \\
\hline $\mathrm{R}$ & ND & $+1-$ & $\begin{array}{l}\text { Aumenta la capacidad de encapsulación del medicamento. Adecuado para la } \\
\text { liberación sostenida y controlada de R. }\end{array}$ & 35 \\
\hline \multicolumn{5}{|c|}{ Nano/micropartículas (NMPs, N-Mcs, NSP) (Figura 1F) } \\
\hline $\mathrm{R}$ & ND & $+/-$ & La NP mejoró la absorción de R en macrófagos. & 36 \\
\hline $\mathrm{H}$ & Oral & $+/+$ & Se mejoró la biodisponibilidad de H y se mantiene una liberación de hasta 24 h. & 37 \\
\hline R y Mxf & ND & $+/-$ & $\begin{array}{l}\text { Las NP promovieron una respuesta inmunológica en macrófagos alveolares } \\
\text { murinos }\end{array}$ & 38 \\
\hline Bdq & ND & $+/-$ & Liberación sostenida de Bdq por 3 días. & 39 \\
\hline Lfx, Cfx, Ofx & ND & $+/-$ & Las NP de Lfx tienen rápida liberación en las primeras $5 \mathrm{~h}$. & 40 \\
\hline $\mathrm{Z}$ & ND & $+/-$ & $\begin{array}{l}\text { Las NP tienen buena captación por los macrófagos alveolares y mantienen una } \\
\text { liberación bifásica: rápida en las primeras } 11 \mathrm{~h} \text { y lenta hasta } 24 \mathrm{~h} \text { que cambia en } \\
\text { relación con el tipo y concentración del polímero. }\end{array}$ & 41 \\
\hline R y H & Inhalatoria & $+/+$ & $\begin{array}{l}\text { Las NP mostraron una menor citotoxicidad y una buena función micobactericida } \\
\text { en los pulmones en comparación con el medicamento libre. }\end{array}$ & 42 \\
\hline
\end{tabular}

* Las estructuras de estas nanopartículas se presenta en la Figura 1E y $1 \mathrm{~F}$.

VA: vía de administración; PC: preclínico; ND: sin datos; Lfx: levofloxacino; Cfx: ciprofloxacino; Ofx: ofloxacino; R: rifampicina; H: isoniazida; Mxf: moxifloxacino, Bdq: bedaquilina; Z: pirazinamida; NP: nanopartícula. 
Tabla 5. Microesferas, nanosuspensiones y nanomicelas utilizadas como transportadoras de fármacos antituberculosos*.

\begin{tabular}{|c|c|c|c|c|}
\hline Fármaco & VA & $\begin{array}{l}\text { In vitro } \\
\text { /PC }\end{array}$ & Comentarios & Ref. \\
\hline \multicolumn{5}{|c|}{ Microesferas (N-Mcs) (Figura 1G) } \\
\hline $\mathrm{R}$ & ND & $+/-$ & $\begin{array}{l}\text { Las NP cargadas con R son absorbidas por las células NR-8383, dentro de las } \\
\text { cuales interactúan con el fagolisosoma y después se degradan. }\end{array}$ & 43 \\
\hline $\mathrm{R}$ & Inhalatoria & $+/-$ & Las NP mostraron liberación constante del fármaco durante 5 días. & 44 \\
\hline $\mathrm{R}$ & Inhalatoria & $+/+$ & Las NP son captadas por macrófagos alveolares y aumentan la captación de R. & 45 \\
\hline \multicolumn{5}{|c|}{ Nano-suspensiones (NSP) (Figura 1H) } \\
\hline Cfx & Inhalatoria & $+/-$ & Presenta una eficiencia del $80 \%-95 \%$ para transportar el medicamento. & 46 \\
\hline \multicolumn{5}{|c|}{ Nanomicelas (NMC) (Figura 1I) } \\
\hline $\mathrm{R}$ & Oral & $+/-$ & $\begin{array}{l}\text { La autoagregación de la } \mathrm{R} \text { en medio acuoso se redujo al mínimo gracias a su } \\
\text { encapsulación en micelas polimérica. }\end{array}$ & 47 \\
\hline $\mathrm{R}$ & ND & $+/-$ & $\begin{array}{l}\text { La liberación de la } \mathrm{R} \text { fue mayor al cambiar un pH ácido (5.3). Las micelas } \\
\text { fueron absorbidas por las líneas celulares A549. }\end{array}$ & 48 \\
\hline R y H & ND & $+/-$ & $\begin{array}{l}\text { Las micelas cargadas de } \mathrm{R} / \mathrm{H} \text { tienen menor toxicidad hemolítica que el me- } \\
\text { dicamento libre. }\end{array}$ & 49 \\
\hline $\mathrm{R}$ & ND & $+1-$ & $\begin{array}{l}\text { Las micelas poliméricas aumentan la concentración intracelular de } \mathrm{R} \text { en los } \\
\text { macrófagos. }\end{array}$ & 50 \\
\hline $\mathrm{R}$ & ND & $+/-$ & $\begin{array}{l}\text { Los ligandos de micelas aumentan la concentración intracelular de R en compara- } \\
\text { ción con los medicamentos libres e indujeron una mayor concentración de citoci- } \\
\text { nas Th1. }\end{array}$ & 51 \\
\hline
\end{tabular}

* Las estructuras de estas nanopartículas se presenta en la Figura 1G, $1 \mathrm{H}$ y $1 \mathrm{I}$.

VA: vía de administración; PC: preclínico; ND: sin datos; Cfx: ciprofloxacin; R: rifampicina; H: isoniazida; NP: nanopartícula.

tos vía oral, tópica, parenteral, etc. En el tratamiento antituberculoso, se han evaluado para transportar clofazimina y ciprofloxacina, algunos ejemplos de se pueden encontrar en la Tabla $5^{(43,44)}$.

\section{Nanomicela (NMC)}

Es una micela que van de $10 \mathrm{~nm}$ a $200 \mathrm{~nm}$, con un núcleo interno hidrófobo y una superficie hidrófila. Debido a su biocompatibilidad, estabilidad estructural, alta capacidad de transportar medicamentos en su superficie externa y de solubilizar medicamentos hidrofóbicos en su núcleo interno, tienen ventajas sobre otras NP para transportar diversos tipos de fármacos. Detalles sobre la evaluación de estas NP para transportar rifampicina se puede encontrar en la Tabla $5^{(45-49)}$.

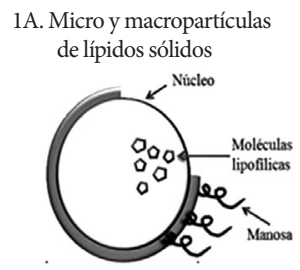

1B. Nanopartículas basadas en emulsiones

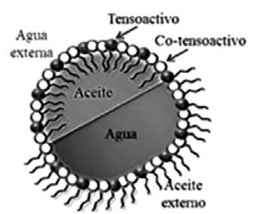

1E. Dendrimeros

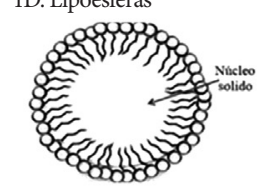

1G. Microesferas

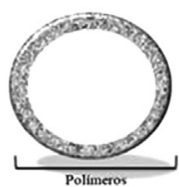

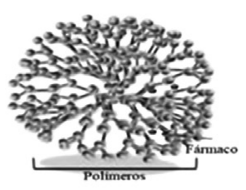

1H. Nano-suspensión

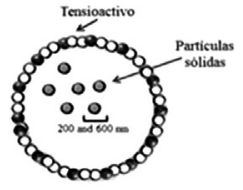

1C. Liposonas/Niosoma

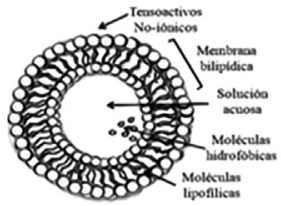

1F. Nano/micropartículas

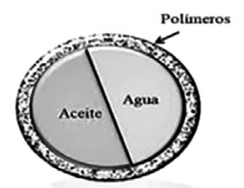

1I. Nanomicelas

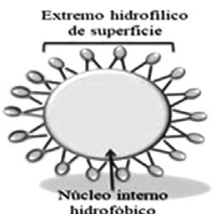

Figura 1. Estructura de nanopartículas empleadas como transportadoras de fármacos contra la Tuberculosis 


\section{NANOFIBRAS}

Las nanofibras comprenden un grupo de materiales estructurados con fibras de tamaño $\leq 100 \mathrm{~nm}$ que se disponen para conformar apósitos de tamaño variable, dependiendo de la aplicación futura. Ofrecen diversas propiedades, como su amplia superficie de contacto que promueve una mejor liberación de los fármacos embebidos, la posibilidad de funcionalización de la superficie, tener una porosidad graduable y el amplio rango de selección de materiales para su elaboración ${ }^{(50)}$. El conjunto de estas propiedades indican que las nanofibras poseen gran potencial para diversos tipos de aplicaciones biomédicas, incluida la liberación de fármacos ${ }^{(51)}$. Actualmente, las técnicas de producción de nanofibras son variadas, siendo el electrohilado la más utilizada ${ }^{(52)}$. El uso de nanofibras para el tratamiento de la TB u otras micobacteriosis es un campo fértil de investigación, se han incorporado fármacos como estreptomicina, isoniacida, pirazinamida y claritromicina con resultados variables ${ }^{(53)}$. Sin embargo, unas películas diseñadas para la administración oral de isoniacida demostraron una alta eficiencia de desintegración, liberación y encapsulamiento del fármaco ${ }^{(54)}$. La utilización de nanofibras también se ha propuesto en la innovación de las técnicas de diagnóstico de TB, por ejemplo, se han preparado nanofibras como biosensores electroquímicos para la detección de ADN de Mycobacterium tuberculosis, las nanofibras resultantes presentaron una alta sensibilidad de detección ${ }^{(55)}$

\section{IMPLICACIONES, BENEFICIOS Y RIESGOS ASOCIADOS AL EMPLEO DE NANOPARTÍCULAS Y NANOMATERIALES EN EL TRATAMIENTO DE TB Y FORMAS RESISTENTES}

Como se mencionó, la resistencia a fármacos antituberculosos y sus formas agravadas, MDR-TB y XDRTB, comprometen el escenario epidemiológico actual y futuro de la TB ${ }^{(56)}$. En este sentido, las NP podrían emplearse como herramientas antituberculosas novedosas para luchar en contra de la TB, transportando una o más combinaciones de medicamentos de primera o segunda línea, e inclusive de nueva generación, usando múltiples vías de aplicación y permitiendo el desarrollo de tratamientos individualizados, según el perfil de resistencia de la micobacteria.

Adicionalmente, estas partículas pueden dirigirse contra un órgano o tejido específico, y considerando que la mico- bacteria se encuentra localizada preferentemente en el pulmón, la posibilidad de dirigir NP a este órgano incrementaría la eficacia del tratamiento ${ }^{(56)}$. Algunas NP tienen la habilidad de transportar simultáneamente fármacos hidrofílicos e hidrofóbicos, manteniendo su integridad, incrementando su biodisponibilidad y eficiencia del tratamiento. Finalmente, las NP también pueden diseñarse para liberar el fármaco de una manera controlada y dosificarlo por periodos prolongados, esto mejoraría la adherencia al tratamiento y disminuiría el riesgo de abandono y desarrollo de resistencia a la TB.

Cabe mencionar que, existen ciertas desventajas asociadas con las NP que requieren revisión. Las NP poseen procesos laboriosos para su formulación, por lo que el escalado para su producción masiva presentaría algunas dificultades técnicas. También se reporta que las NP poseen una considerable tendencia a la agregación, este factor podría tener efectos colaterales no deseados, como una trombosis vascular, lo cual generaría afecciones dermatológicas y cardiovasculares. Estos efectos se han observado al emplear NP como transportadoras de antígenos ${ }^{(12)}$.

\section{CONCLUSIONES}

Sin duda, el empleo de NP abre un campo totalmente novedoso para la administración de esquemas de fármacos para el tratamiento de la $\mathrm{TB}$, esto puede tener implicancias en su manejo y de esta manera apoyar en el cumplimiento del tratamiento y éxito de la curación de esta enfermedad.

Asimismo, la mayoría de los estudios relacionados con el uso y la evaluación de NP y nanomateriales contra la TB se han realizado en ensayos in vitro y con menor frecuencia en ensayos in vivo (Tablas 1-5). No fue posible encontrar un reporte que describiera algún ensayo clínico que evalúe alguna NP en el contexto de experimentación en humanos. Entonces, es evidente que aún resta mucho por investigar y determinar en términos de inocuidad y bioseguridad, antes de garantizar beneficios reales de estas nuevas tecnologías. No hay duda que el desarrollo de este campo se intensificará en un futuro próximo.

Contribuciones de autoría: JLH y RZC participaron en la concepción y diseño, el análisis y recuperación de la información, y en la redacción, revisión y aprobación de la versión final. DEPM y VMH colaboraron en el análisis y recuperación de la información, redacción, revisión y aprobación de la versión final.

Financiamiento: JLH fue parcialmente financiada por el Proyecto SIP 20200492, del Instituto Politécnico Nacional y es becaria del SNI, EDI y COFAA. DEPM es becario del CONACyT n.o 411155.

Conflictos de interés: Los autores declaran no tener conflictos de interés.

Material suplementario: Disponible en la versión electrónica de la RPMESP. 


\section{REFERENCIAS BIBLIOGRÁFICAS}

1. World Health Organizaiton. 2015 tuberculosis Global report. World Heal Organ Geneva. [Internet]. WHO; 2015 [cited November 20, 2019]. Disponible en: https://www.who.int/tb/publications/global_report/ gtbr2015_executive_summary.pdf?ua

2. Transforming Our World: The 2030 Agenda for Sustainable Development. In: William Rosa MS. A New Era in Global Health. New York, NY: Springer Publishing Company; 2018. doi: 10.1891/9780826190123.ap02.

3. Moreno-Molina M, Comas I, Furió V.The Future of TB Resistance Diagnosis: The Essentials on Whole Genome Sequencing and Rapid Testing Methods. Arch Bronconeumol. 2019;55(8):421-426. doi: 10.1016/j.arbres.2019.01.002.

4. Sugawara E, Nikaido H. Properties of AdeABC and AdeIJK Efflux Systems of Acinetobacter baumannii Compared with Those of the AcrAB-TolC System of Escherichia coli. Antimicrob Agents Chemother. 2014;58(12):7250-7257. doi: 10.1128/AAC.03728-14.

5. Li Y, Sun F, Zhang W. Bedaquiline and delamanid in the treatment of multidrug-resistant tuberculosis: Promising but challenging. Drug Dev Res. 2018;80(1):ddr.21498. doi: 10.1002/ddr.21498.

6. Piddock LJ V. Reflecting on the final report of the O'Neill Review on Antimicrobial Resistance. Lancet Infect Dis. 2016;16(7):767-768. doi: 10.1016/S1473-3099(16)30127-X.

7. O’ Neil J. Review on Antibiotic Resisitance. Antimicrobial Resistance: Tackling a Crisis for the Health and Wealth of Nations [Internet] 2014 [cited November 20, 2019]. Disponible en: https://amr-review.org/sites/ default/files/AMR\%20Review\%20Paper\%20-\%20Tackling\%20a\%20crisis\%20for\%20the\%20health\%20and\%20wealth\%20of\%20nations_1.pdf

8. Xu K, Liang ZC, Ding X, Hu H, Liu S, Nurmik M, et al. Nanomaterials in the Prevention, Diagnosis, and Treatment of Mycobacterium Tuberculosis Infections. Adv Healthc Mater. 2018;7(1):1700509. doi: 10.1002/adhm.201700509.

9. Andrade F, Rafael D, Videira M, Ferreira D, Sosnik A, Sarmento B. Nanotechnology and pulmonary delivery to overcome resistance in infectious diseases. Adv Drug Deliv Rev. 2013;65(13-14):18161827. doi: 10.1016/j.addr.2013.07.020.

10. Grotz E, Tateosian N, Amiano N, Cagel M, Bernabeu E, Chiappetta DA, et al. Nanotechnology in Tuberculosis: State of the Art and the Challenges Ahead. Pharm Res. 2018;35(11):213. doi: 10.1007/s11095-018-2497-z.

11. Singh J, Garg T, Rath G, Goyal AK. Advances in nanotechnology-based carrier systems for targeted delivery of bioactive drug molecules with special emphasis on immunotherapy in drug resistant tuberculosis - a critical review. Drug Deliv. 2016;23(5):1676-1698. doi: 10.3109/10717544.2015.1074765.

12. El-Sayed A, Kamel M. Advances in nanomedical applications: diagnostic, therapeutic, immunization, and vaccine production. Environ Sci Pollut Res. 2019. doi: 10.1007/s11356-019-06459-2.

13. Singh H, Jindal S, Singh M, Sharma G, Kaur IP. Nano-formulation of rifampicin with enhanced bioavailability: Development, characterization and in-vivo safety. Int J Pharm. 2015;485(1-2):138-151. doi: 10.1016/j. ijpharm.2015.02.050.

14. Vieira ACC, Chaves LL, Pinheiro S, Pinto S, Pinheiro M, Costa Lima S, et al. Mucoadhesive chitosan-coated solid lipid nanoparticles for better management of tuberculosis. Int J Pharm. 2018;536(1):478-485. doi: 10.1016/j.ijpharm.2017.11.071.

15. Maretti E, Costantino L, Rustichelli C, Leo E, Croce MA, Buttini F, et al. Surface engineering of Solid Lipid Nanoparticle assemblies by methyl $a-d$-mannopyranoside for the active targeting to macrophages in anti-tuberculosis inhalation therapy. Int J Pharm. 2017;528(1-2):440-451. doi: 10.1016/j.ijpharm.2017.06.045.

16. Costa A, Sarmento B, Seabra V. Mannose-functionalized solid lipid nanoparticles are effective in targeting alveolar macrophages. Eur J Pharm Sci. 2018;114:103-113. doi: 10.1016/j.ejps.2017.12.006.
17. Chuan J, Li Y, Yang L, Sun X, Zhang Q, Gong T, et al. Enhanced rifampicin delivery to alveolar macrophages by solid lipid nanoparticles. J Nanoparticle Res. 2013;15(5):1634. doi: 10.1007/s11051-013-1634-1.

18. De Matteis L, Jary D, Lucía A, García-Embid S, Serrano-Sevilla I, Pérez $\mathrm{D}$, et al. New active formulations against $\mathrm{M}$. tuberculosis: Bedaquiline encapsulation in lipid nanoparticles and chitosan nanocapsules. Chem Eng J. 2018;340:181-191. doi: 10.1016/j.cej.2017.12.110.

19. Bhardwaj A, Mehta S, Yadav S, Singh SK, Grobler A, Goyal AK, et al. Pulmonary delivery of antitubercular drugs using spray-dried lipidpolymer hybrid nanoparticles. Artif Cells, Nanomedicine, Biotechnol. 2016;44(6):1544-1555. doi: 10.3109/21691401.2015.1062389.

20. Song X, Lin Q, Guo L, Fu Y, Han J, Ke H, et al. Rifampicin Loaded Mannosylated Cationic Nanostructured Lipid Carriers for Alveolar Macrophage-specific Delivery. Pharm Res. 2015;32(5):1741-1751. doi: 10.1007/s11095-014-1572-3.

21. Kaur G, Mehta SK. Probing Location of Anti-TB Drugs Loaded in Brij 96 Microemulsions Using Thermoanalytical and Photophysical Approach. J Pharm Sci. 2014;103(3):937-944. doi: 10.1002/jps.23857.

22. Mehta SK, Kaur G, Bhasin KK. Entrapment of multiple anti-Tb drugs in microemulsion system: Quantitative analysis, stability, and in vitro release studies. J Pharm Sci. 2010;99(4):1896-1911. doi: 10.1002/jps.21971.

23. Manca ML, Sinico C, Maccioni AM, Diez O, Fadda AM, Manconi M. Composition influence on pulmonary delivery of rifampicin liposomes. Pharmaceutics. 2012;4(4):590-606. doi: 10.3390/pharmaceutics4040590.

24. Gaidukevich SK, Mikulovich YL, Smirnova TG, Andreevskaya SN, Sorokoumova GM, Chernousova LN, et al. Antibacterial Effects of Liposomes Containing Phospholipid Cardiolipin and Fluoroquinolone Levofloxacin on Mycobacterium tuberculosis with Extensive Drug Resistance. Bull Exp Biol Med. 2016;160(5):675-678. doi: 10.1007/s10517-016-3247-z.

25. Patil J, Devi Vk, Devi K, Sarasija S. A novel approach for lung delivery of rifampicin-loaded liposomes in dry powder form for the treatment of tuberculosis. Lung India. 2015;32(4):331. doi: 10.4103/0970-2113.159559.

26. Bhardwaj A, Kumar L, Narang RK, Murthy RSR. Development and characterization of ligand-appended liposomes for multiple drug therapy for pulmonary tuberculosis. Artif Cells, Nanomedicine, Biotechnol. 2013;41(1):52-59. doi: 10.3109/10731199.2012.702316.

27. Rojanarat W, Nakpheng T, Thawithong E, Yanyium N, Srichana T. Inhaled pyrazinamide proliposome for targeting alveolar macrophages. Drug Deliv. 2012;19(7):334-345. doi: 10.3109/10717544.2012.721144.

28. El-Ridy MS, Yehia SA, Kassem MA-E-M, Mostafa DM, Nasr EA, Asfour MH. Niosomal encapsulation of ethambutol hydrochloride for increasing its efficacy and safety. Drug Deliv. 2015;22(1):21-36. doi: 10.3109/10717544.2013.868556.

29. El-Ridy MS, Abdelbary A, Nasr EA, Khalil RM, Mostafa DM, El-Batal AI, et al. Niosomal encapsulation of the antitubercular drug, pyrazinamide. Drug Dev Ind Pharm. 2011;37(9):1110-1118. doi: 10.3109/03639045.2011.560605.

30. Mehta SK, Jindal N. Formulation of Tyloxapol niosomes for encapsulation, stabilization and dissolution of anti-tubercular drugs. Colloids Surfaces B Biointerfaces. 2013;101:434-441. doi: 10.1016/j.colsurfb.2012.07.006.

31. Singh G, Dwivedi H, SarafS, Saraf S. Niosomal Delivery of Isoniazid - Development and Characterization. Trop J Pharm Res. 2011;10(2):203-210. doi: 10.4314/tjpr.v10i2.66564.

32. Takenaga M, Ohta Y, Tokura Y, Hamaguchi A, Igarashi R, Disratthakit A, et al. Lipid Microsphere Formulation Containing Rifampicin Targets Alveolar Macrophages. Drug Deliv. 2008;15(3):169-175. doi: 10.1080/10717540801952530.

33. Vijayaraj Kumar P, Agashe H, Dutta T, Jain N. PEGylated Dendritic Architecture for Development of a Prolonged Drug Delivery System for an Antitubercular Drug. Curr Drug Deliv. 2007;4(1):11-19. doi: 10.2174/156720107779314794. 
34. Trousil J, Filippov SK, Hrubý M, Mazel T, Svyrová Z, Cmarko D, et al. System with embedded drug release and nanoparticle degradation sensor showing efficient rifampicin delivery into macrophages. Nanomedicine Nanotechnology, Biol Med. 2017;13(1):307-315. doi: 10.1016/j. nano.2016.08.031

35. Gajendiran M, Gopi V, Elangovan V, Murali RV, Balasubramanian S. Isoniazid loaded core shell nanoparticles derived from PLGA-PEG-PLGA tri-block copolymers: In vitro and in vivo drug release. Colloids Surfaces B Biointerfaces. 2013;104:107-115. doi: 10.1016/j.colsurfb.2012.12.008

36. Sarfraz M, Shi W, Gao Y, Clas S-D, Roa W, Bou-Chacra N, et al. Immune response to antituberculosis drug-loaded gelatin and polyisobutyl-cyanoacrylate nanoparticles in macrophages. Ther Deliv. 2016;7(4):213-228. doi: 10.4155/tde-2015-0007.

37. Ritsema JAS, Herschberg EMA, Borgos SE, Løvmo C, te Welscher YM, Storm G, et al. Relationship between polarities of antibiotic and polymer matrix on nanoparticle formulations based on aliphatic polyesters. Int J Pharm. 2018;548(2):730-739. doi: 10.1016/j.ijpharm.2017.11.017.

38. Cheow WS, Hadinoto K. Factors affecting drug encapsulation and stability of lipid-polymer hybrid nanoparticles. Colloids Surfaces B Biointerfaces. 2011;85(2):214-220. doi: 10.1016/j.colsurfb.2011.02.033.

39. Ravi Varma J, Kumar Ts, Prasanthi B, Ratna Jv. Formulation and characterization of pyrazinamide polymeric nanoparticles for pulmonary tuberculosis: Efficiency for alveolar macrophage targeting. Indian J Pharm Sci. 2015;77(3):258. doi: 10.4103/0250-474X.159602.

40. Garg T, Rath G, Goyal AK. Inhalable chitosan nanoparticles as antitubercular drug carriers for an effective treatment of tuberculosis. Artif Cells, Nanomedicine, Biotechnol. 2015;44(3):1-5. doi: 10.3109/21691401.2015.1008508.

41. Onoshita T, Shimizu Y, Yamaya N, Miyazaki M, Yokoyama M, Fujiwara $\mathrm{N}$, et al. The behavior of PLGA microspheres containing rifampicin in alveolar macrophages. Colloids Surfaces B Biointerfaces. 2010;76(1):151157. doi: 10.1016/j.colsurfb.2009.10.036.

42. Doan TVP, Olivier JC. Preparation of rifampicin-loaded PLGA microspheres for lung delivery as aerosol by premix membrane homogenization. Int J Pharm. 2009;382(1-2):61-66. doi: 10.1016/j.ijpharm.2009.08.008.

43. Ohashi K, Kabasawa T, Ozeki T, Okada H. One-step preparation of rifampicin/poly(lactic-co-glycolic acid) nanoparticle-containing mannitol microspheres using a four-fluid nozzle spray drier for inhalation therapy of tuberculosis. J Control Release. 2009;135(1):19-24. doi: 10.1016/j. jconrel.2008.11.027.

44. El-Gendy N, Desai V, Berkland C. Agglomerates of Ciprofloxacin Nanoparticles Yield Fine Dry Powder Aerosols. J Pharm Innov. 2010;5(3):79-87. doi: 10.1007/s12247-010-9082-2.

45. Grotz E, Bernabeu E, Pappalardo M, Chiappetta DA, Moretton MA Nanoscale Kolliphor ${ }^{\circledR}$ HS 15 micelles to minimize rifampicin self-aggregation in aqueous media. J Drug Deliv Sci Technol. 2017;41:1-6. doi: 10.1016/j.jddst.2017.06.009
46. Praphakar RA, Munusamy MA, Rajan M. Development of extended-voyaging anti-oxidant Linked Amphiphilic Polymeric Nanomicelles for Anti-Tuberculosis Drug Delivery. Int J Pharm. 2017;524(1-2):168-177. doi: 10.1016/j.ijpharm.2017.03.089.

47. Upadhyay S, Khan I, Gothwal A, Pachouri PK, Bhaskar N, Gupta UD, et al. Conjugated and Entrapped HPMA-PLA Nano-Polymeric Micelles Based Dual Delivery of First Line Anti TB Drugs: Improved and Safe Drug Delivery against Sensitive and Resistant Mycobacterium Tuberculosis. Pharm Res. 2017;34(9):1944-1955. doi: 10.1007/ s11095-017-2206-3.

48. Moretton MA, Chiappetta DA, Andrade F, das Neves J, Ferreira D, Sarmento B, et al. Hydrolyzed Galactomannan-Modified Nanoparticles and Flower-Like Polymeric Micelles for the Active Targeting of Rifampicin to Macrophages. J Biomed Nanotechnol. 2013;9(6):1076-1087. doi: 10.1166/ jbn.2013.1600.

49. Gao Y, Sarfraz MK, Clas S-D, Roa W, Löbenberg R. Hyaluronic Acid-Tocopherol Succinate-Based Self-Assembling Micelles for Targeted Delivery of Rifampicin to Alveolar Macrophages. J Biomed Nanotechnol. 2015;11(8):1312-1329. doi: 10.1166/jbn.2015.2091.

50. Rasouli R, Barhoum A, Bechelany M, Dufresne A. Nanofibers for Biomedical and Healthcare Applications. Macromol Biosci. 2019;19(2):1800256. doi: 10.1002/mabi.201800256

51. Sill TJ, von Recum HA. Electrospinning: Applications in drug delivery and tissue engineering. Biomaterials. 2008;29(13):1989-2006. doi: 10.1016/j. biomaterials.2008.01.011.

52. Wang X, Ding B, Li B. Biomimetic electrospun nanofibrous structures for tissue engineering. Mater Today. 2013;16(6):229-241. doi: 10.1016/j. mattod.2013.06.005.

53. Hassounah IA, Shehata NA, Kimsawatde GC, Hudson AG, Sriranganathan N, Joseph EG, et al. Studying the activity of antitubercluosis drugs inside electrospun polyvinyl alcohol, polyethylene oxide, and polycaprolacton nanofibers. J Biomed Mater Res Part A. 2014;102(11):4009-4016. doi: 10.1002/jbm.a.35070

54. Chachlioutaki K, Tzimtzimis EK, Tzetzis D, Chang MW, Ahmad Z, Karavasili C, et al. Electrospun Orodispersible Films of Isoniazid for Pediatric Tuberculosis Treatment. Pharmaceutics. 2020;12(5):470. doi 10.3390/pharmaceutics12050470.

55. Mohamad FS, Zaid MHM, Abdullah J, Zawawi RM, Lim HN, Sulaiman Y, et al. Synthesis and Characterization of Polyaniline/Graphene Composite Nanofiber and Its Application as an Electrochemical DNA Biosensor for the Detection of Mycobacterium tuberculosis. Sensors. 2017;17(12):2789. doi: 10.3390/s17122789.

56. Dheda K, Gumbo T, Maartens G, Dooley KE, Murray M, Furin J, et al. The Lancet Respiratory Medicine Commission: 2019 update: epidemiology, pathogenesis, transmission, diagnosis, and management of multidrug-resistant and incurable tuberculosis. Lancet Respir Med. 2019;7(9):820-826. doi: 10.1016/S2213-2600(19)30263-2. 